\title{
COMUNICAÇÃO ENTRE A BURSA SINOVIAL DO OSSO NAVICULAR E A ARTICULAÇÃO INTERFALANGEANA DISTAL EM EQÜINOS
}

\author{
COMMUNICATION BETWEEN THE SYNOVIAL BURSA OF THE NAVICULAR BONE AND \\ THE DISTAL INTERPHALANGEAL JOINT IN EQUINES
}

\author{
Edson Moreira Borges ${ }^{1}$ Maria Verônica de Souza ${ }^{2}$ \\ Tarcisio Antônio Rego de Paula ${ }^{3}$
}

RESUMO

\begin{abstract}
Os bloqueios anestésicos são importantes no diagnóstico de claudicação em eqüinos, e também uma alternativa viável para anestesias em uma variedade de procedimentos. Com o objetivo de avaliar a existência ou não de comunicação entre a bursa sinovial do osso navicular e a articulação interfalangeana distal, foram utilizados 9 cavalos mestiços, nos quais foi administrado $3 \mathrm{ml}$ de lipoidol $50 \%$ na bursa sinovial do osso navicular. Em $33 \%$ dos casos observou-se contraste na região dorsal da articulação interfalangeana distal após administração deste na bursa sinovial do osso navicular, o que sinaliza esta comunicação, sendo que a mesma ocorreu apenas nos animais adultos.
\end{abstract}

Palavras-chave: bursa sinovial do osso navicular, anestesia intra-articular, osso navicular, articulação interfalangeana distal.

\section{SUMMARY}

The anaesthesic blocks are important in the diagnose of lameness in equines, and an alternative to anaesthesias in several procedures. In this experiment, nine crossbred equines were utilized. It was given $3 \mathrm{ml}$ lipoidol $50 \%$ in the navicular bone synovial bursa in all animals, with the purpose of evaluating the existence of communication between the navicular bone synovial bursa and distal interphalangeal joint. In 33,3\% of the cases it was observed contrast in the dorsal region of the distal interphalangeal joint after its administration in the synovial bursa of the navicular bone. The communication occured only in the adult animals.

Key words: navicular bone, synovial bursa, intra-articular anaesthesia, distal interfalangeal joint.

\section{INTRODUÇÃO}

Bloqueios anestésicos são bastante utilizados na prática veterinária como meio diagnóstico de claudicação em eqüinos. Espera-se que, após a execução desses bloqueios, o animal deixe de claudicar e apoie temporariamente o membro comprometido, desde que a lesão seja dessensibilizada (DYSON, 1984, 1986; GIBSON \& STASHAK, 1989; KEG $\boldsymbol{e t}$ al., 1992). Além da técnica de bloqueios nervosos utilizada como meio de diagnóstico de claudicação em eqüino, a anestesia intra-articular e intra-bursal são também utilizadas para localizar a área lesionada, particularmente quando achados radiográficos não são conclusivos.

A articulação interfalangeana distal (AID) e a bursa sinovial do osso navicular (BN) são cavidades sinoviais de interesse clínico e acadêmico por serem

\footnotetext{
${ }^{1}$ Médico Veterinário, aluno do Curso de Especialização da Universidade Federal de Viçosa (UFV).

${ }^{2}$ Médico Veterinário, Professora Assistente, Departamento de Veterinária, Área de Clínica de Grandes Animais, UFV, 36571-000, Viçosa, MG. Autor para correspondência.

${ }^{3}$ Médico Veterinário, Professor Assistente, UFV.
} 
locais freqüentes de alterações clínicas (BOWKER $\boldsymbol{e} t$ al., 1993; GIBSON et al., 1990). O bloqueio da BN é bastante utilizado quando se suspeita de problemas relacionados ao osso navicular, principalmente na sindrome do navicular ou podotrocleose que é uma das causas mais comuns de claudicação crônica em eqüinos. A bursa sinovial do osso navicular encontrase entre o tendão do músculo flexor digital profundo (TFDP) e o osso sesamóide distal (osso navicular; GIBSON et al., 1990), sendo o seu bloqueio realizado no aspecto palmar do membro torácico ou pélvico, em um ponto intermediário aos bulbos dos talões, na base da fossa digital. Inicialmente administra-se $1 \mathrm{ml}$ de anestésico local subcutaneamente e em seguida introduz-se a agulha no mesmo local e penetra-se dorsalmente até encontrar o osso sesamóide distal. Nesse ponto, a bolsa foi penetrada. A agulha deverá ser levemente tracionada e $5 \mathrm{ml}$ de anestésico deverá ser depositado (STASHAK, 1994).

MOYER (1989) comenta a incerteza de que o material administrado na BN permanece sem se comunicar com a AID ou com a bainha do tendão do músculo flexor digital profundo. Comunicação entre a AID e a bainha do tendão do músculo flexor digital profundo tem sido observada em potros, mas não em cavalos adultos, após utilização de injeções de latex (CALISTAR \& St CLAIR, 1969). Estes mesmos autores ainda estudando a interrelação entre a bursa sinovial do osso navicular e articulação interfalangeana distal não observaram comunicação entre estas duas estruturas tanto nos animais mais jovens quanto nos mais velhos, porém os autores acreditam que em algum momento, mesmo que de forma temporária, possa ocorrer comunicação entre as duas estruturas pelo fato de bursas e cápsulas articulares serem cavitações do tecido conjuntivo embrionário, ou seja, por terem a mesma origem.

GIBSON et al. (1990) realizaram um estudo administrando $3 \mathrm{ml}$ de contraste a base de iodo na bursa sinovial do osso navicular em 50 membros torácicos, sendo 13 cavalos vivos e 24 peças frescas de cadáveres adultos, para determinar a freqüência de comunicação entre a bursa sinovial do osso navicular e a cápsula articular da articulação interfalangeana distal. Os autores acreditam que provavelmente não exista comunicação natural entre a BN e a AID.

Uma resposta positiva para anestesia da $\mathrm{BN}$ pode refletir na presença de bursite navicular (TUNER, 1989) ou doença do navicular (STASHAK, 1994), excetuando-se no caso em que a última evolua para a excessiva formação de aderência entre o tendão do músculo flexor digital profundo (TFDP) e o osso navicular (STASHAK, 1994). Neste caso o problema passa a ser mecânico e não responde ao bloqueio anestésico.

Para determinar se a AID se comunicava direta ou indiretamente com a BN, BOWKER $\boldsymbol{e t}$ al. (1993), infiltraram 3ml de corante na BN ou $5 \mathrm{ml}$ na AID. O corante foi diluído em solução salina a $0,9 \%$ (sendo em 90 dígitos na AID e em 28 dígitos na $\mathrm{BN}$ ), mepivacaína (em 26 dígitos na AID) ou látex (sendo em 6 dígitos na AID e em 2 dígitos na $\mathrm{BN}$ ), totalizando 152 dígitos obtidos de eqüinos e pôneis durante necropsia. Dos 122 membros que receberam corante na AID, $120(98,4 \%)$ não demonstraram evidência de comunicação com a BN. Dos 30 dígitos submetidos a injeções na BN, 14 (46,7\%) tinham vestígio de corante no tecido conjuntivo dorsal ao ligamento sesamoideano colateral, e dessa forma, foram descartados do estudo. Nos demais 16 dígitos (53,3\%), a difusão do corante foi limitada a BN, especialmente na porção proximal da mesma, com exceção de 2 dígitos que tinham evidência de comunicação direta entre a BN e a AID.

Estudos a respeito de soluções radiopacas (GIBSON et al., 1990) mostraram que essas substâncias contêm componentes de alto peso molecular e com pouca chance de se difundir para a membrana sinovial ou outro tecido conectivo dessa região.

Como pode ser constatado, há controvérsias quanto a presença ou não de comunicação entre a bursa sinovial do osso navicular e a cápsula articular da articulação interfalangeana distal. Esse fato levanta dúvidas quanto a especificidade no diagnóstico de anormalidades envolvendo o osso sesamóide distal e falange distal.

Este trabalho tem como objetivo, observar a existência de comunicação entre a bursa sinovial do osso navicular e a cápsula articular da articulação interfalangeana distal através da infiltração de contraste radiopaco.

\section{MATERIAIS E MÉTODOS}

Foram utilizados nove cavalos, clinicamente sadios, sendo seis fêmeas e três machos, sem raça definida, com idade entre 16 meses a 13 anos, com peso corporal variando entre 165 e $530 \mathrm{~kg}$, provenientes da microrregião de Viçosa e setor de equideocultura do Departamento de Zootecnia da Universidade Federal de Viçosa - MG.

Em cada animal, mantido em estação e o membro torácico flexionado, foi administrado $3 \mathrm{ml}$ de lipoidol $^{\text {a }}$ (contraste), com auxílio de uma agulha medindo $6 \mathrm{~cm}$ de comprimento e 1,2 de diâmetro, no interior da bursa sinovial do osso navicular. A injeção 
do contraste foi realizada na face palmar do membro direito e posteriormente no membro esquerdo, em todos os animais. Para isso o local foi tricotomizado, lavado com sabão neutro e preparado antisepticamente com iodo-povidine. A agulha foi direcionada dorsalmente através da pele entre os bulbos dos talões (GIBSON et al., 1990; Figura 1). Para que os animais aceitassem mais facilmente a execução da técnica, foi administrada detomidinab na dose de $10 \mu \mathrm{g} / \mathrm{kg}$, por via intra-venosa, antes da infiltração do contraste.

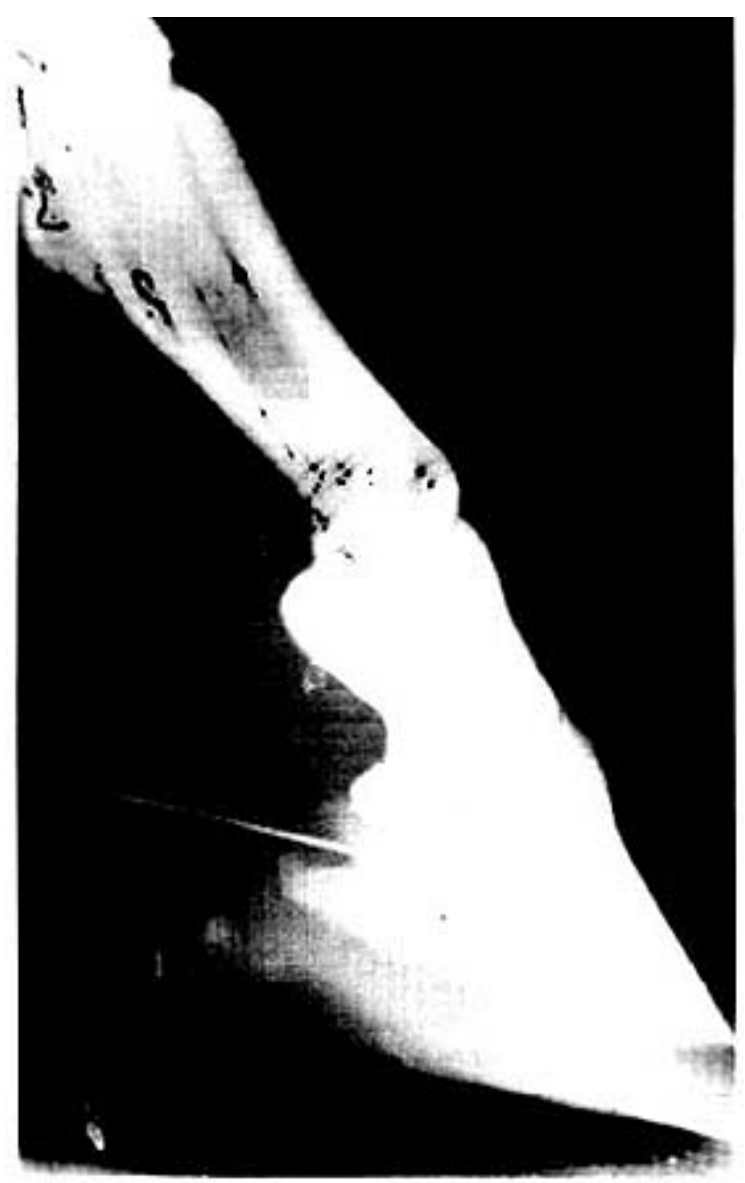

Figura 1 - Incal de infiltrayalo de contrastc na bursa sinovial do osso naviculas đo membro torácico equino.

Com a finalidade de se determinar o grau de difusão do contraste na BN e AID, foram realizadas radiografias látero-mediais na extremidade distal de ambos os membros torácicos. As radiografias foram feitas com o animal em estação, antes e imediatamente após da administração do contraste. Não houve locomoção dos animais no espaço compreendido entre a administração do contraste e a execução do exame radiológico.
Terminada a técnica, todos os animais receberam fenilbutazona ${ }^{c}$ por via intra-venosa, na dose de $4,4 \mathrm{mg} / \mathrm{kg}$ de peso vivo, a cada 12 horas, durante três dias e $2,2 \mathrm{mg} / \mathrm{kg}$ a cada 12 horas, por mais dois dias para evitar um possível processo inflamatório. Após o tratamento, os eqüinos foram mantidos em observação por mais três dias e finalmente foram liberados. Os animais de número 3 e 6 apresentaram claudicação e foram adequadamente tratados até o desaparecimento do quadro clínico. O tratamento utilizado para esses animais foi o mesmo citado anteriormente, com adição de ducha fria e compressa de água quente, durante três dias. Não houve necessidade de se prolongar o tratamento, pois o mesmo foi suficiente para o desaparecimento do quadro clínico. Os dados observados neste experimento foram tabulados para comunicação entre a bursa sinovial do osso navicular e a articulação interfalangeana distal.

\section{RESULTADOS E DISCUSSÃO}

Neste estudo observou-se que $33,3 \%$ dos animais apresentaram contraste na região dorsal da articulação interfalangeana distal, o que sugere comunicação entre a bursa sinovial do osso navicular e a articulação interfalangeana distal, após infiltração de contraste na BN (Figura 2). Estes animais tinham idades que variavam entre 120 a 156 meses, ou seja, já eram adultos. Nos mais jovens não se observou a passagem do contraste (Tabela 1), o que difere dos achados encontrados por CALISTAR \& St. CLAIR (1969).

Acredita-se, portanto, que o fato dos animais mais velhos apresentarem peso superior aos mais novos pode estar diretamente relacionado com a presença de comunicação entre as duas estruturas. Ainda como uma possível hipótese da comunicação entre a bursa sinovial do osso navicular e a cápsula articular da articulação interfalangeana distal, existe o fato desses animais serem mais velhos e estarem sujeitos a um maior número de lesões por concussão e trauma nos membros torácicos, com possibilidade de formação de fístula entre a BN e a AID.

Segundo GIBSON et al. (1990), provavelmente não ocorra comunicação natural entre a BN e a AID. Neste experimento no entanto, houve contato entre as duas estruturas em 33,3\% dos animais, mesmo seguindo-se as recomendações de STASHAK (1994). A dose utilizada foi a mesma em todos os animais, assim como a pressão na seringa durante a administração do contraste.

Diante dos achados deste experimento, acredita-se que o bloqueio da bursa sinovial do osso 
navicular poderá, em alguns casos, dessensibilizar não apenas a bursa sinovial do osso navicular, como também o tendão do músculo flexor digital profundo ou o osso sesamóide distal, como mencionam alguns autores (STASHAK, 1994; TUNER, 1989), mas também a articulação interfalangeana distal.

Tabela I - Presença ou tusẻncia de comunicaçio entre a bursa sinovial do osso navicular e a cápsula articułar da articulacio interfalangeana distal. no memhos torécico diresto e esquerde de equitino.

\begin{tabular}{|c|c|c|c|c|c|}
\hline \multirow{2}{*}{$\begin{array}{l}N^{0} \text { do } \\
\text { animal }\end{array}$} & \multirow{2}{*}{$\begin{array}{l}\text { Idade } \\
\text { (meses) }\end{array}$} & \multirow[t]{2}{*}{ Sexo } & \multirow{2}{*}{$\begin{array}{l}\text { Peso } \\
\mathrm{kg}\end{array}$} & MTD & MTE \\
\hline & & & & ('comunica & nmuni \\
\hline
\end{tabular}

\begin{tabular}{cccccc}
\hline I & 120 & M & 400 & - & + \\
2 & 156 & M & 310 &. & + \\
3 & 120 & M & 530 & + & + \\
4 & 30 & F & 260 & - & - \\
5 & 26 & F & 270 &. &. \\
6 & 24 & F & 205 & &. \\
7 & 16 & F & 217 &. &. \\
8 & 22 & F & 205 & - & - \\
9 & 20 & 1 & 165 &. &. \\
\hline
\end{tabular}

MTD: Membro toracico direiro: MTE: Membro rorácico esquerdo; * prescnça de comunicnçío entro a bursu sinuvial do osso navicular $\mathrm{c}$ a anticulaçso interfalangesanta distal. - : ausencia de comunicacto entre a bursa sinovial do osso navicular c a cápsula articulas da articulaçðo interfalangeana discal: F: Fenca: M: Macho

O fato dos animais terem apresentado comunicação entre a $\mathrm{BN}$ e a AID não pode ser relacionado exclusivamente com a questão da idade e peso, pois como pode ser constatado na Tabela 1, a comunicação ocorreu apenas em animais adultos e do sexo masculino. Assim, como todos os machos eram adultos e todas as fêmeas jovens, tal comportamento também pode estar relacionado ao sexo, o que neste experimento não foi possível ser verificado, uma vez que não houve a disponibilidade de animais machos jovens, nem de fêmeas adultas para a realização deste estudo. Desta forma, novos trabalhos, com um número maior de animais, de ambos os sexos e de variadas idades, deverão ser conduzidos no sentido da confirmação da tendência observada no presente trabalho, bem como permitir conclusões mais precisas.

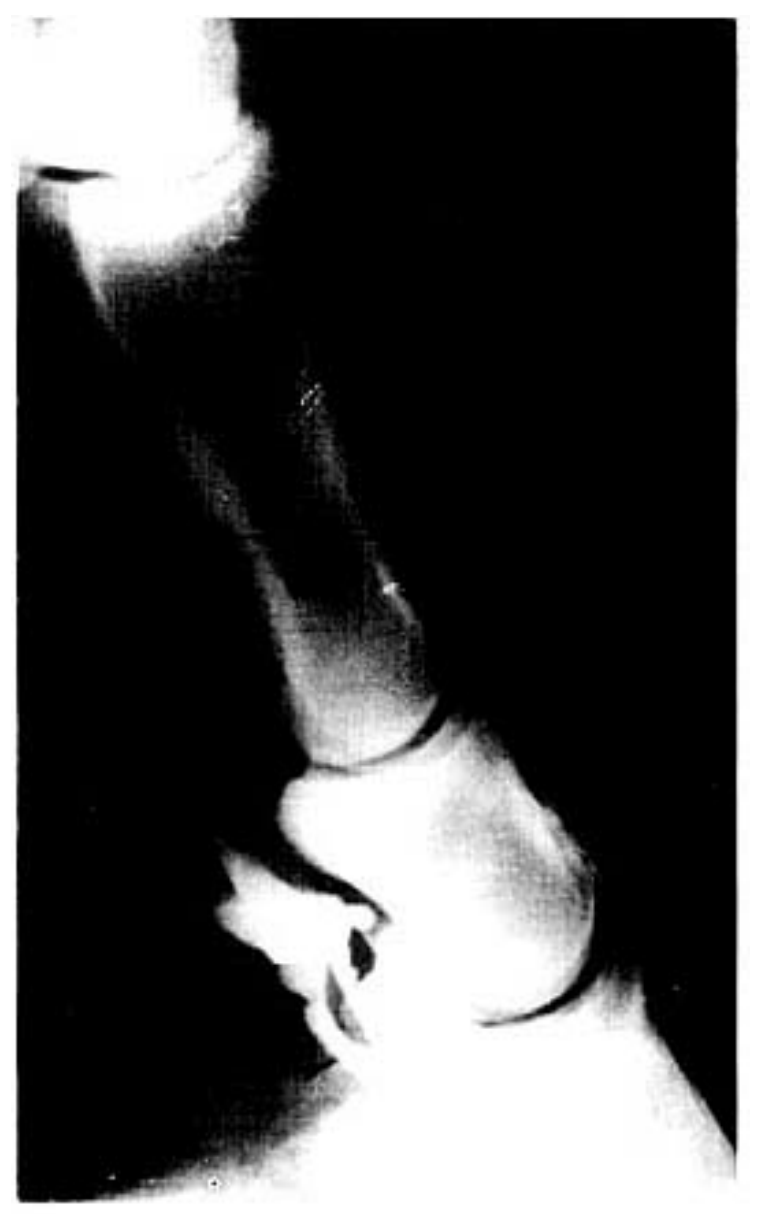

Figura 2 - Prescnça de contraste na regiåo dorsal da articulą̧ăo interfalangeana distal (scta) após infiltraç̧o dc contraste na hursa sinovial do osso navicular (cabeça de sets) tos mernbru torácios esquerdo do cavalo $n .1$.

\section{CONCLUSÕES}

Nos eqüinos adultos pode ocorrer comunicação entre a bursa sinovial do osso navicular e a cápsula articular da articulação interfalangeana distal, após administração de contraste na bursa sinovial do osso navicular.

Com base na administração do contraste, o bloqueio da bursa sinovial do osso navicular não é específico como meio de diagnóstico de alterações envolvendo o osso sesamóide distal e sua bursa sinovial.

\section{FONTES DE AQUISIÇÃO}

a - Hypaque 50\%: Winthrop Products Inc. Av. Brasil, 22155, RJ. b - Domosedan: Ciba-Geigy Química S.A. Div. Agric. SubDivisão de Saúde Animal, Av. S ${ }^{\text {to }}$ Amaro, 5137, SP. Caixa postal 21468. 
c - Equipalazone: Marcolab Industria de Produtos Veterinários Ltda. R.C, 35 Jardim Primavera, Duque de Caxias, RJ.

\section{AGRADECIMENTOS}

A CAPES e ao Hospital Veterinário do Departamento de Veterinária da Universidade Federal de Viçosa (DVT/UFV) pelo suporte financeiro e ao setor de equideocultura do Departamento de Zootecnia da Universidade Federal de Viçosa pelo empréstimo dos animais.

Um agradecimento especial ao funcionário Claudio Roberto Fialho (DVT/UFV) pelo apoio técnico.

\section{REFERÊNCIAS BIBLIOGRÁFICAS}

BOWKER, R.M., ROCKERSHOUSER, S. J., VEX, K. B. et al Immunocytochemical and dye distribution studies of nerves potentially desensitized by injections into the distal interphalangeal joint or the navicular bursa of horses. J Am Vet Med Assoc, v. 203, n. 12, p. 1708-1714, 1993.

CALISLAR, T., St CLAIR, L.E. Observations on the navicular bursa and the distal interphalangeal joint cavity in the horse. J Am Vet Assoc, v. 154, p. 410-412, 1969.

DYSON, S. Nerve blocks and lameness diagnosis in the horse. In-Practice, v. 6, n. 4, p. 102-107, 1984.
DYSON, S. Problems associated with the interpretation of the results of regional and intra-articular anaesthesia in the horse. Vet Rec, v. 118, p. 419-422, 1986.

GIBSON, K.T., McILWRAITH, C.W., PARK, R.D. A radiographic study of the distal interphalangeal joint and navicular bursa of the horse. Vet Radiol, v. 3, n. 1, p. 22-25, 1990.

GIBSON, K.T., STASHAK, T.S. Using perineural anesthesia to localize equine lameness. Vet Med, v. 84, n. 11, p. 1082, 10841086, 1989.

KEG, P.R., BELT, A.J.M. Van den., MERKENS, H.W. et al. The effect of regional nerve blocks on the lameness caused by collagenase induced tendonitis in the midmetacarpal region of the horse: a study using gait analysis and ultrassonography to determine tendon healing. J Vet Med, v. 39, n. 5, p. 349363,1992

MOYER, W. Clinical examination of the equine foot. Vet Clin North Am Equine Pract,v. 5, n. 1, p. 29-46, 1989.

STASHAK, T. Claudicação em eqüinos segundo Adams. São Paulo: Roca, 1994. Cap. 3: Diagnóstico de claudicação: p. 99-159.

TUNER, T. Diagnosis and treatment of navicular syndrome in horses. Vet Clin North Am Equine Pract, v. 5, p. 131-144, 1989. 